QUARTERLY OF APPLIED MATHEMATICS

VOLUME LXVII, NUMBER 4

DECEMBER 2009, PAGES 661-685

S 0033-569X(09)01134-2

Article electronically published on September 2, 2009

\title{
THE EULER-POINCARÉ THEORY OF METAMORPHOSIS
}

\author{
BY \\ DARRYL D. HOLM (Department of Mathematics, Imperial College London SW7 2AZ, United \\ Kingdom and Computer and Computational Science, Los Alamos National Laboratory, MS D413 Los \\ Alamos, New Mexico 87545), \\ ALAIN TROUVÉ (CMLA (CNRS, URA 1611), Ecole Normale Supérieure de Cachan, 61, avenue du \\ Président Wilson, F-94 235 Cachan Cedex), \\ AND \\ LAURENT YOUNES (Center for Imaging Science, The Johns Hopkins University, 3400 N-Charles \\ Street, Baltimore, Maryland 21218-2686)
}

\begin{abstract}
In the pattern matching approach to imaging science, the process of "metamorphosis" is template matching with dynamical templates (Trouvé and Younes, Found. Comp. Math., 2005). Here, we recast the metamorphosis equations of that paper into the Euler-Poincaré variational framework of Holm, Marsden, and Ratiu, Adv. in Math., 1998 and show that the metamorphosis equations contain the equations for a perfect complex fluid (Holm, Springer, 2002). This result connects the ideas underlying the process of metamorphosis in image matching to the physical concept of an order parameter in the theory of complex fluids. After developing the general theory, we reinterpret various examples, including point set, image and density metamorphosis. We finally discuss the issue of matching measures with metamorphosis, for which we provide existence theorems for the initial and boundary value problems.
\end{abstract}

1. Overview. Pattern matching is an important component of imaging science, with privileged applications in computerized anatomical analysis of medical images (computational anatomy) [2, 4, 12, 23. When comparing images, the purpose is to find, based on the conservation of photometric cues, an optimal nonrigid alignment between the images.

Received April 27, 2008.

2000 Mathematics Subject Classification. Primary 58E50.

Key words and phrases. Groups of diffeomorphisms, EPDiff, image registration, shape analysis, deformable templates.

The work of D. D. Holm was partially supported by the US Department of Energy, Office of Science, Applied Mathematical Research, and the Royal Society of London Wolfson Research Merit Award. D. D. Holm is grateful for stimulating discussions with C. Tronci.

The work of Laurent Younes was partially supported by NSF DMS-0456253.

E-mail address: d.holm@ic.ac.uk, dholm@lanl.gov

E-mail address: trouve@cmla.ens-cachan.fr

E-mail address: laurent.younes@jhu.edu

(C)2009 Brown University 
In this context, diffeomorphic pattern matching methods have been developed, based on this principle, and on the additional goal of defining a (Riemannian) metric structure on spaces of deformable objects [7, 24]. They have found multiple applications in medical imaging [16, 3, 11, 30, 10, 29], where the objects of interest include images, landmarks, measures (modeling unlabeled point sets) and currents (modeling curves and surfaces). These methods address the registration problem by solving a variational problem of the form

$$
\text { Find } \arg \min \left(d(\mathrm{id}, g)^{2}+\operatorname{Errorterm}\left(g \cdot n_{\text {temp }}, n_{\text {targ }}\right)\right)
$$

over all diffeomorphisms $g$, where $n_{\text {temp }}$ and $n_{\text {targ }}$ are the compared objects (usually referred to as the template and the target), $(g, n) \mapsto g . n$ is the action of diffeomorphisms on the objects and $d$ is a right-invariant Riemannian distance on diffeomorphisms. This problem therefore directly relates to geodesics in groups of diffeomorphisms, namely to the EPDiff equation [1, 18, 14, and the conserved initial momentum that specifies the solution has been used in statistical studies in order to provide anatomical characterizations of mental disorders [22, 31].

One of the issues in problems formulated as (11) is that the error term breaks the metric aspects inherited from the distance $d$ on diffeomorphisms. This model has an inherent template vs. target asymmetry, which is not always justified. With the purpose of designing a fully metric approach to the template matching problem, Metamorphoses have been introduced in [20, and formalized and studied in [28, 27]. They provide interesting pattern matching alternatives to (1), in a completely metric framework. In this paper, we pursue the following twofold goal: (i) provide a generic Lagrangian formulation for metamorphoses that includes the Riemannian formalism introduced in [28, and (ii) study a new form of metamorphoses, adapted to the deformation of measures.

We start with (i), for which our point of view will be fairly abstract. We consider a manifold, $N$, which is acted upon by a Lie group $G: N$ contains what we can refer to as "deformable objects" and $G$ is the group of deformations, which is the group of diffeomorphisms in our applications. We will review several examples for the space $N$ later on in this paper.

Definition 1. A metamorphosis 28 is a pair of curves $\left(g_{t}, \eta_{t}\right) \in G \times N$ parameterized by time $t$, with $g_{0}=$ id. Its image is the curve $n_{t} \in N$ defined by the action $n_{t}=g_{t} \cdot \eta_{t}$. The quantities $g_{t}$ and $\eta_{t}$ are called the deformation part of the metamorphosis, and its template part, respectively. When $\eta_{t}$ is constant, the metamorphosis is said to be a pure deformation. In the general case, the image is a combination of a deformation and template variation.

In 28, metamorphoses were used to modify an original Riemannian metric on $N$ by including a deformation component in the geodesic evolution. In this paper, we generalize the approach to a generic Lagrangian formulation, and apply the Euler-Poincaré variational framework [14 to derive evolution equations. More specific statements on these equations (for example, regarding the existence and uniqueness of solutions) require additional assumptions on $G$ and the space $N$ of deformed objects. In the second part of this paper, we will review the case in which $N$ is a space of linear forms on a 
Hilbert space of smooth functions, which will allow us to define metamorphoses between measures.

The next section provides the notation and definitions related to the general problem of metamorphoses.

2. Notation and Lagrangian formulation. We will use either letters $\eta$ or $n$ to denote elements of $N$, the former being associated to the template part of a metamorphosis, and the latter to its image.

The variational problem we shall study optimizes over metamorphoses $\left(g_{t}, \eta_{t}\right)$ by minimizing, for some Lagrangian $L$,

$$
\int_{0}^{1} L\left(g_{t}, \dot{g}_{t}, \eta_{t}, \dot{\eta}_{t}\right) d t
$$

with fixed boundary conditions for the initial and final images $n_{0}$ and $n_{1}$ (with $n_{t}=g_{t} \eta_{t}$ ) and $g_{0}=\mathrm{id}_{G}$ (so only the images are constrained at the end-points, with the additional normalization $g_{0}=$ id).

Let $\mathfrak{g}$ denote the Lie algebra of $G$. We will consider Lagrangians defined on $T G \times T N$ that satisfy the following invariance condition: there exists a function $\ell$ defined on $\mathfrak{g} \times T N$ such that

$$
L\left(g, U_{g}, \eta, \xi_{\eta}\right)=\ell\left(U_{g} g^{-1}, g \eta, g \xi_{\eta}\right) .
$$

In other terms, $L$ is invariant by the right action of $G$ on $G \times N$ defined by $(g, \eta) h=$ $\left(g h, h^{-1} \eta\right)$.

For a metamorphosis $\left(g_{t}, \eta_{t}\right)$, we therefore have, letting $u_{t}=\dot{g}_{t} g_{t}^{-1}, n_{t}=g_{t} \eta_{t}$ and $\nu_{t}=g_{t} \dot{\eta}_{t}$,

$$
L\left(g_{t}, \dot{g}_{t}, \eta_{t}, \dot{\eta}_{t}\right)=\ell\left(u_{t}, n_{t}, \nu_{t}\right)
$$

The Lie derivative with respect to a vector field $X$ will be denoted $\mathcal{L}_{X}$. The Lie algebra of $G$ is identified with the set of right invariant vector fields $U_{g}=u g, u \in T_{\mathrm{id}} G=\mathfrak{g}$, $g \in G$, and we will use the notation $\mathcal{L}_{u}=\mathcal{L}_{U}$.

The Lie bracket $[u, v]$ on $\mathfrak{g}$ is defined by

$$
\mathcal{L}_{[u, v]}=-\left(\mathcal{L}_{u} \mathcal{L}_{v}-\mathcal{L}_{v} \mathcal{L}_{u}\right)
$$

and the associated adjoint operator is $\operatorname{ad}_{u} v=[u, v]$. Letting $I_{g}(h)=g h g^{-1}$ and $A d_{v} g=$ $\mathcal{L}_{v} I_{g}(\mathrm{id})$, we also have $\operatorname{ad}_{u} v=\mathcal{L}_{u}\left(\operatorname{Ad}_{v}\right)(\mathrm{id})$. When $G$ is a group of diffeomorphisms, this yields $a d_{u} v=D u v-D v u$.

The pairing between a linear form $l$ and a vector $u$ will be denoted $(l \mid u)$. Duality with respect to this pairing will be denoted with a $*$ exponent.

When $G$ acts on a manifold $\tilde{N}$, the $\diamond$ operator is defined on $T \tilde{N}^{*} \times \tilde{N}$ and takes values in $\mathfrak{g}^{*}$. It is defined by

$$
(\delta \diamond \tilde{n} \mid u)=-(\delta \mid u \tilde{n})
$$


3. Euler equations. We compute the Euler equations associated with the minimization of

$$
\int_{0}^{1} \ell\left(u_{t}, n_{t}, \nu_{t}\right) d t
$$

with fixed boundary conditions $n_{0}$ and $n_{1}$. We therefore consider variations $\delta u$ and $\omega=\delta n$. The variation $\delta \nu$ can be obtained from $n=g \eta$ and $\nu=g \dot{\eta}$ yielding $\dot{n}=\nu+u n$ and $\dot{\omega}=\delta \nu+u \omega+\delta u n$. Here and in the remainder of this paper, we assume that computations are performed in a local chart on $T N$ with respect to which we take partial derivatives.

We therefore have

$$
\int_{0}^{1}\left(\left(\frac{\delta \ell}{\delta u} \mid \delta u_{t}\right)+\left(\frac{\delta \ell}{\delta n} \mid \omega_{t}\right)+\left(\frac{\delta \ell}{\delta \nu} \mid \dot{\omega}_{t}-u_{t} \omega_{t}-\delta u_{t} n_{t}\right)\right) d t=0 .
$$

The $\delta u$ term yields the equation

$$
\frac{\delta \ell}{\delta u}+\frac{\delta \ell}{\delta \nu} \diamond n_{t}=0
$$

(Note the abuse of notation: $\delta \ell / \delta \nu \in T(T N)^{*}$ is considered as a linear form on $T N$ by $(\delta \ell / \delta \nu \mid z):=(\delta \ell / \delta \nu \mid(0, z))$.) For the $\omega$ term, we get, after an integration by parts,

$$
\frac{\partial}{\partial t} \frac{\delta \ell}{\delta \nu}+u_{t} \star \frac{\delta \ell}{\delta \nu}-\frac{\delta \ell}{\delta n}=0
$$

where we have used the notation

$$
\left(\frac{\delta \ell}{\delta \nu} \mid u \omega\right)=\left(u \star \frac{\delta \ell}{\delta \nu} \mid \omega\right)
$$

We therefore obtain the system of equations

$$
\left\{\begin{array}{l}
\frac{\delta \ell}{\delta u}+\frac{\delta \ell}{\delta \nu} \diamond n_{t}=0 \\
\frac{\partial}{\partial t} \frac{\delta \ell}{\delta \nu}+u_{t} \star \frac{\delta \ell}{\delta \nu}=\frac{\delta \ell}{\delta n} \\
\dot{n}_{t}=\nu_{t}+u_{t} n_{t}
\end{array}\right.
$$

Note that $\frac{\delta \ell}{\delta u}+\frac{\delta \ell}{\delta \nu} \diamond n$ is the momentum arising from Noether's theorem for the invariance of the Lagrangian being considered. The special form of the boundary conditions (fixed $n_{0}$ and $n_{1}$ ) ensures that this momentum is zero.

4. Euler-Poincaré reduction. An equivalent system can be obtained via an EulerPoincaré reduction [14. In this setting, we make the variation in the group element and in the template instead of the velocity and the image. We let $\xi_{t}=\delta g_{t} g_{t}^{-1}$ and $\varpi_{t}=g_{t} \delta \eta_{t}$. From this, we obtain the expressions of $\delta u, \delta n$ and $\delta \nu$. We first have $\delta u_{t}=\dot{\xi}_{t}+\left[\xi_{t}, u_{t}\right]$; this comes from the standard Euler-Poincaré reduction theorem, as provided in [14, 18]. We also have $\delta n_{t}=\delta\left(g_{t} \eta_{t}\right)=\varpi_{t}+\xi_{t} n_{t}$. From $\nu_{t}=g_{t} \dot{\eta}_{t}$, we get $\delta \nu_{t}=g_{t} \delta \dot{\eta}_{t}+\xi_{t} \nu_{t}$ and from $\varpi_{t}=g_{t} \delta \eta_{t}$ we also have $\dot{\varpi}_{t}=u_{t} \varpi_{t}+g_{t} \dot{\eta}_{t}$. This yields $\delta \nu_{t}=\dot{\varpi}_{t}+\xi_{t} \nu_{t}-u_{t} \varpi_{t}$. 
We also compute the boundary conditions for $\xi$ and $\varpi$. At $t=0$, we have $g_{0}=$ id and $n_{0}=g_{0} \eta_{0}=\mathrm{cst}$, which implies $\xi_{0}=0$ and $\varpi_{0}=0$. At $t=1$, the relation $g_{1} \eta_{1}=\mathrm{cst}$ yields $\xi_{1} n_{1}+\omega_{1}=0$.

Now, the first variation is

$$
\int_{0}^{1}\left(\left(\frac{\delta \ell}{\delta u} \mid \dot{\xi}_{t}-\operatorname{ad}_{u_{t}} \xi_{t}\right)+\left(\frac{\delta \ell}{\delta n_{t}} \mid \varpi_{t}+\xi_{t} n_{t}\right)+\left(\frac{\delta \ell}{\delta \nu} \mid \dot{\varpi}_{t}+\xi_{t} \nu_{t}-u_{t} \varpi_{t}\right)\right) d t=0 .
$$

In the integration by parts to eliminate $\dot{\xi}_{t}$ and $\dot{\varpi}_{t}$, the boundary term is $\left((\delta \ell / \delta u)_{1} \mid \xi_{1}\right)+$ $\left((\delta \ell / \delta \nu)_{1} \mid \omega_{1}\right)$. Using the boundary condition, the last term can be rewritten as

$$
-\left((\delta \ell / \delta \nu)_{1} \mid \xi_{1} n_{1}\right)=\left((\delta \ell / \delta \nu)_{1} \diamond n_{1} \mid \xi_{1}\right) .
$$

We therefore obtain the boundary equation

$$
\frac{\delta \ell}{\delta u}(1)+\frac{\delta \ell}{\delta \nu}(1) \diamond n_{1}=0 .
$$

The evolution equation for $\xi$ is

$$
\frac{\partial}{\partial t} \frac{\delta \ell}{\delta u}+\operatorname{ad}_{u_{t}}^{*} \frac{\delta \ell}{\delta u}+\frac{\delta \ell}{\delta n} \diamond n_{t}+\frac{\delta \ell}{\delta \nu} \diamond \nu_{t}=0
$$

and the one for $\varpi$ is

$$
\frac{\partial}{\partial t} \frac{\delta \ell}{\delta \nu}+u_{t} \star \frac{\delta \ell}{\delta \nu}-\frac{\delta \ell}{\delta n}=0 .
$$

We therefore obtain the system

$$
\left\{\begin{array}{l}
\frac{\partial}{\partial t} \frac{\delta \ell}{\delta u}+\operatorname{ad}_{u_{t}}^{*} \frac{\delta \ell}{\delta u}+\frac{\delta \ell}{\delta n} \diamond n_{t}+\frac{\delta \ell}{\delta \nu} \diamond \nu_{t}=0 \\
\frac{\partial}{\partial t} \frac{\delta \ell}{\delta \nu}+u_{t} \star \frac{\delta \ell}{\delta \nu}-\frac{\delta \ell}{\delta n}=0 \\
\frac{\delta \ell}{\delta u}(1)+\frac{\delta \ell}{\delta \nu}(1) \diamond n_{1}=0 \\
\dot{n}_{t}=\nu_{t}+u_{t} n_{t} .
\end{array}\right.
$$

The system (5) is equivalent to (4), since they characterize the same critical points. Direct evidence of this fact can be obtained by rewriting the first equation in (5) in the form:

$$
\frac{\partial}{\partial t}\left(\frac{\delta \ell}{\delta u}+\frac{\delta \ell}{\delta \nu} \diamond u\right)+\operatorname{ad}_{u_{t}}^{*}\left(\frac{\delta \ell}{\delta u}+\frac{\delta \ell}{\delta \nu} \diamond u\right)=0 .
$$

We indeed have, for a solution of (5),

$$
\begin{aligned}
\frac{\partial}{\partial t}\left(\frac{\delta \ell}{\delta u_{t}}+\frac{\delta \ell}{\delta \nu} \diamond n_{t}\right) & =\frac{\partial}{\partial t} \frac{\delta \ell}{\delta u}+\left(\frac{\partial}{\partial t} \frac{\delta \ell}{\delta \nu}\right) \diamond n_{t}+\frac{\delta \ell}{\delta \nu} \diamond \dot{n}_{t} \\
& =\frac{\partial}{\partial t} \frac{\delta \ell}{\delta u}+\left(\frac{\delta \ell}{\delta n}-u_{t} \star \frac{\delta \ell}{\delta \nu}\right) \diamond n_{t}+\frac{\delta \ell}{\delta \nu} \diamond\left(\nu_{t}+u_{t} n_{t}\right) \\
& =\frac{\partial}{\partial t} \frac{\delta \ell}{\delta u}+\frac{\delta \ell}{\delta n} \diamond n_{t}+\frac{\delta \ell}{\delta \nu} \diamond \nu_{t}-\left(u_{t} \star \frac{\delta \ell}{\delta \nu}\right) \diamond n_{t}+\frac{\delta \ell}{\delta \nu} \diamond\left(u_{t} n_{t}\right) \\
& =-\operatorname{ad}_{u_{t}}^{*} \frac{\delta \ell}{\delta u}-\operatorname{ad}_{u_{t}}^{*}\left(\frac{\delta \ell}{\delta \nu} \diamond n_{t}\right) .
\end{aligned}
$$


In the last equation, we have used the fact that, for any $\alpha \in \mathfrak{g}$,

$$
\begin{aligned}
\left(\frac{\delta \ell}{\delta \nu} \diamond(u n)-\left(u \star \frac{\delta \ell}{\delta \nu}\right) \diamond n \mid \alpha\right) & =\left(\frac{\delta \ell}{\delta \nu} \mid \alpha(u n)-u(\alpha n)\right) \\
& =-\left(\frac{\delta \ell}{\delta \nu} \mid[u, \alpha] n\right) \\
& =-\left(\frac{\delta \ell}{\delta \nu} \diamond n \mid[u, \alpha]\right) \\
& =-\left(\operatorname{ad}_{u}^{*}\left(\frac{\delta \ell}{\delta \nu} \diamond n\right) \mid \alpha\right) .
\end{aligned}
$$

This equation, combined with $(\delta \ell / \delta u)_{1}+(\delta \ell / \delta \nu)_{1} \diamond u_{1}=0$, obviously implies the first equation in (4).

\section{Special cases.}

5.1. Riemannian metric. A primary application of this framework can be based on the definition of a Riemannian metric on $G \times N$ which is invariant for the action of $G$ : $(g, \eta) h=\left(g h, h^{-1} \eta\right)$, the corresponding Lagrangian then taking the form

$$
l(u, n, \nu)=\|(u, \nu)\|_{n}^{2} .
$$

The variational problem is now equivalent to the computation of geodesics for the canonical projection of this metric from $G \times N$ onto $N$. This construction has been introduced in [20]. The evolution equations have been derived and studied in [28] in the case $l(u, n, \nu)=|u|_{\mathfrak{g}}^{2}+|\nu|_{n}^{2}$, for a given norm, $|\cdot|_{\mathfrak{g}}$, on $\mathfrak{g}$ and a pre-existing Riemannian structure on $N$.

The interest of this construction is that this provides a Riemannian metric on $N$ which incorporates the group actions. Examples of this are given below for point sets and images.

5.2. Semi-direct product. Assume that $N$ is a group and that for all $g \in G$, the action of $g$ on $N$ is a group homomorphism: For all $n, \tilde{n} \in N, g(n \tilde{n})=(g n)(g \tilde{n})$ (for example, $N$ can be a vector space and the action of $G$ can be linear). Consider the semi-direct product $G$ S $N$ with $(g, n)(\tilde{g}, \tilde{n})=(g \tilde{g},(g \tilde{n}) n)$ and build on $G$ S $N$ a right-invariant metric constrained by its value \|\|$_{\left(\mathrm{id}_{G}, \mathrm{id}_{N}\right)}$ at the identity. Then, optimizing the geodesic energy in $G$ S $N$ between $\left(\operatorname{id}_{G}, n_{0}\right)$ and $\left(g_{1}, n_{1}\right)$ with fixed $n_{0}$ and $n_{1}$ and free $g_{1}$ yields a particular case of metamorphoses.

Right invariance for the metric on $G(S)$ implies

$$
\left\|\left(U_{g}, \zeta\right)\right\|_{(g, n)}=\|\left(U_{g} \tilde{g},\left(U_{g} \tilde{n}\right) n+(g \tilde{n}) \zeta \|_{(g \tilde{g},(g \tilde{n}) n)},\right.
$$

which, using $(\tilde{g}, \tilde{n})=\left(g^{-1}, g^{-1} n^{-1}\right)$, yields, letting $u=U_{g} g^{-1}$,

$$
\begin{aligned}
\left\|\left(U_{g}, \zeta\right)\right\|_{(g, n)} & =\|\left(u,\left(u n^{-1}\right) n+n^{-1} \zeta \|_{\left(\operatorname{id}_{G}, \operatorname{id}_{N}\right)}\right. \\
& =\|\left(u, n^{-1}(\zeta-u n) \|_{\left(\operatorname{id}_{G}, \operatorname{id}_{N}\right)}\right.
\end{aligned}
$$

since $0=u\left(n^{-1} n\right)=\left(u n^{-1}\right) n+n^{-1}(u n)$. So, the geodesic energy on $G \mathrm{~S} N$ for a path of length 1 is

$$
\int_{0}^{1} \|\left(u_{t}, n_{t}^{-1}\left(\dot{n}_{t}-u_{t} n_{t}\right) \|_{\left(\mathrm{id}_{G}, \mathrm{id}_{N}\right)}^{2}\right.
$$


and optimizing this with fixed $n_{0}$ and $n_{1}$ is equivalent to solving the metamorphosis problem with

$$
l(u, n, \nu)=\left\|\left(u, n^{-1} \nu\right)\right\|_{\left(\mathrm{id}_{G}, \mathrm{id}_{N}\right)}^{2} .
$$

This turns out to be a particular case of the previous example. The situation is even simpler when $N$ is a vector space with additive group operation, since this implies (identifying all tangent spaces on $N) n^{-1} \nu=\nu$ for all $n$ and the Lagrangian does not depend on $n$, which gives a very simple form to systems (4) and (5). They become

$$
\left\{\begin{array}{l}
\frac{\delta \ell}{\delta u}+\frac{\delta \ell}{\delta \nu} \diamond n_{t}=0 \\
\frac{\partial}{\partial t} \frac{\delta \ell}{\delta \nu}+u_{t} \star \frac{\delta \ell}{\delta \nu}=0 \\
\dot{n}_{t}=\nu_{t}+u_{t} n_{t}
\end{array}\right.
$$

and

$$
\left\{\begin{array}{l}
\frac{\partial}{\partial t} \frac{\delta \ell}{\delta u}+\operatorname{ad}_{u_{t}}^{*} \frac{\delta \ell}{\delta u}+\frac{\delta \ell}{\delta \nu} \diamond \nu_{t}=0 \\
\frac{\partial}{\partial t} \frac{\delta \ell}{\delta \nu}+u_{t} \star \frac{\delta \ell}{\delta \nu}=0 \\
\frac{\delta \ell}{\delta u}(1)+\frac{\delta \ell}{\delta \nu}(1) \diamond n_{1}=0 \\
\dot{n}_{t}=\nu_{t}+u_{t} n_{t} .
\end{array}\right.
$$

Even when $N$ is not a vector space, metamorphoses obtained from the semi-direct product formulation are specific among general metamorphoses, because they satisfy the conservation of momentum property which comes with every Lie group with a rightinvariant metric. This conservation equation can be written

$$
\left(\frac{\delta \ell}{\delta u}, \frac{\delta \ell}{\delta \nu}\right)_{t}=A d_{\left(g_{t}, n_{t}\right)^{-1}}^{*}\left(\frac{\delta \ell}{\delta u}, \frac{\delta \ell}{\delta \nu}\right)_{t=0}
$$

where the adjoint representation is the one associated to the semi-direct product. This property (which we do not make explicit in the general case) will be illustrated in some of the examples below.

5.3. Constrained metamorphoses. Returning to the general formulation, it is sometimes useful to include constraints on $n_{t}$, the image of the metamorphosis, making the minimization in (2) subject to $\Phi\left(n_{t}\right)=0(t \in[0,1])$ for some function $\Phi: N \rightarrow \mathbb{R}^{q}$. 
Using Lagrange multipliers, this directly provides a new version of (4), yielding

$$
\left\{\begin{array}{l}
\frac{\delta \ell}{\delta u}+\frac{\delta \ell}{\delta \nu} \diamond n_{t}=0, \\
\frac{\partial}{\partial t} \frac{\delta \ell}{\delta \nu}+u_{t} \star \frac{\delta \ell}{\delta \nu}=\frac{\delta \ell}{\delta n}-\lambda_{t}^{T} \frac{\delta \Phi}{\delta n} \\
\dot{n}_{t}=\nu_{t}+u_{t} n_{t}, \\
\left(\frac{\delta \Phi}{\delta n} \mid \dot{n}_{t}\right)=0 .
\end{array}\right.
$$

6. Examples from pattern matching. In the following examples and in the rest of the paper, $G$ is a group of diffeomorphisms over some open subset $\Omega \subset \mathbb{R}^{d}$. We will assume that elements of $G$ can be obtained as flows associated to ordinary differential equations of the form $\dot{g}_{t}=u_{t} \circ g_{t}$, where $u_{t}$ is assumed to belong, at all times, to a Hilbert space $\mathfrak{g}$ of vector fields on $\Omega$ with the condition

$$
\int_{0}^{1}\left\|u_{t}\right\|_{\mathfrak{g}}^{2} d t<\infty
$$

We will assume that elements of $\mathfrak{g}$ are smooth enough, namely that $V$ can be continuously embedded in the space of $C^{p}$ vector fields with vanishing $p$ first derivatives on $\partial \Omega$ and at infinity, for some $p \geq 1$. More details in this construction can be found in 27] (Appendix C).

We will write the inner product in $\mathfrak{g}$ in the form $\langle u, v\rangle_{V}=\left(L_{\mathfrak{g}} u \mid v\right)$, where $L_{\mathfrak{g}}$ is the duality operator from $\mathfrak{g}$ to $\mathfrak{g}^{*}$. Its inverse, a kernel operator, will be denoted $K_{\mathfrak{g}}$.

6.1. Landmarks and peakons. The space $N$ contains the objects that are subject to deformations. The simplest case probably corresponds to configurations of $Q$ landmarks, for which $N=\Omega^{Q}$. So elements $\eta, \nu \in H$ are $Q$-tuples of points in $\Omega$, with tangent vectors being $Q$-tuples of $d$-dimensional vectors.

The model that has been proposed in [20, 5] corresponds to the Lagrangian

$$
\ell(u, n, \nu)=\|u\|_{\mathfrak{g}}^{2}+\frac{1}{\sigma^{2}} \sum_{k=1}^{Q}\left|\nu^{(k)}\right|^{2}
$$

This Lagrangian is therefore independent of $n$ (but does not correspond to a semidirect product). We have $\delta \ell / \delta u=2 L_{\mathfrak{g}} u$ and $(\delta \ell / \delta \nu)=\left(2 / \sigma^{2}\right)\left(\nu^{(1)}, \ldots, \nu^{(Q)}\right)$. Let $n=\left(q^{(1)}, \ldots, q^{(Q)}\right)$. From the definition $((\delta \ell / \delta \nu) \diamond n \mid w)=-(\delta \ell / \delta \nu \mid w n)$, we get (since $\left.w n=\left(w\left(q^{(1)}\right), \ldots, w\left(q^{(Q)}\right)\right)\right)$ :

$$
\frac{\delta \ell}{\delta \nu} \diamond n=-2 \sum_{k=1}^{Q} \frac{\nu^{(k)}}{\sigma^{2}} \otimes \delta_{q^{(k)}} .
$$

Here and later, we use the following notation: if $f$ is a vector field on $\mathbb{R}^{d}$ (considered as a vector density) and if $\mu$ is a measure on $\mathbb{R}^{d}$, then the linear form $f \otimes \mu$, acting on 
vector fields, is defined by

$$
(f \otimes \mu \mid w)=\int_{\mathbb{R}^{d}} f(x)^{T} w(x) d \mu .
$$

Our first equation for landmark metamorphosis is therefore

$$
L_{\mathfrak{g}} u_{t}=\sum_{k=1}^{N} \frac{\nu_{t}^{(k)}}{\sigma^{2}} \otimes \delta_{q_{t}^{(k)}} .
$$

The second equation is $\frac{\partial}{\partial t}(\delta \ell / \delta \nu)+u \star(\delta \ell / \delta \nu)=0$, which in this case gives

$$
\dot{\nu}_{t}^{(k)}+D u_{t}\left(q_{t}^{(k)}\right)^{T} \nu_{t}^{(k)}=0, \quad k=1, \ldots, Q .
$$

Introducing $p^{(k)}=\nu^{(k)} / \sigma^{2}$, we can rewrite system (4) in the form:

$$
\left\{\begin{array}{l}
L_{\mathfrak{g}} u_{t}=\sum_{k=1}^{Q} p_{t}^{(k)} \otimes \delta_{q_{t}^{(k)}}, \\
\dot{p}_{t}^{(k)}+D u_{t}\left(q_{t}^{(k)}\right)^{T} p_{t}^{(k)}=0, \quad k=1, \ldots, Q, \\
\dot{q}^{(k)}=u_{t}\left(q_{t}^{(k)}\right)+\sigma^{2} p_{t}^{(k)}, \quad k=1, \ldots, Q .
\end{array}\right.
$$

Putting the evolution equations into this form is interesting because the limiting case, $\sigma^{2}=0$, exactly corresponds to the peakon solution of the EPDiff equation [14, the dynamics of which having recently been described in [19]. It is important to see, however, that the solutions may have significantly distinct behavior when $\sigma>0$. Figures 1 and 2 illustrate this in the case of two landmarks in 1D. The plots show the evolution of $r=q_{2}-q_{1}$ over time when $K_{\mathfrak{g}}=L_{\mathfrak{g}}^{-1}$ is a Gaussian kernel. Figure 1 provides a comparison in the case of a head-on collision $\left(p_{1}+p_{2}=0\right)$. In the case $\sigma^{2}=0$, the peakons approach each other infinitely closely in time without colliding. For positive $\sigma^{2}$, the peakons get close, slow down, then cross over and their distance grows rapidly to infinity. The duration of the collision phase $(q \simeq 0)$ decreases when the sum of the absolute momenta $\left(p_{1}-p_{2}\right)$ increases. Note that the deformation $g_{t}$ never becomes singular during this process. The space first contracts when the peakons get closer, then expands after the crossover.

In Figure2, the case of one peakon overtaking another is shown. In the case $\sigma^{2}=0$, we observe a well-known behavior: the peakons approach very closely, then separate again without crossing over. For metamorphoses $\left(\sigma^{2}>0\right)$, the details of the behavior depend on the initial difference between the momenta. If it is small, then the evolution is similar to the case $\sigma^{2}=0$. When the initial momentum difference becomes larger, the peakon that started behind has enough energy to overpass the other one and the two peakons exchange position. In all cases, the deformations first experience a contraction, then an expansion (relative to the position of the two peakons).

6.2. Images. Now, consider the case when $N$ is a space of smooth functions from $\Omega$ to $\mathbb{R}$, which we will call images, with the action $(g, n) \mapsto n \circ g^{-1}$. A simple case of 

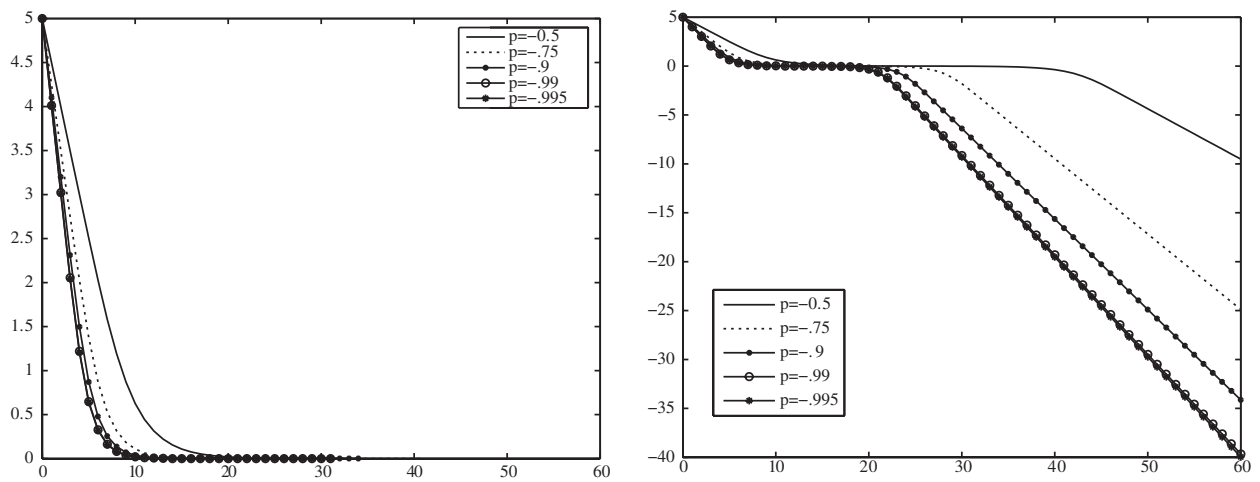

Fig. 1. Head-on collision between two peakons. The plots show the evolution of $q=q_{2}-q_{1}$ over time for several values of $p=p_{2}-p_{1}$, for $\sigma^{2}=0$ (left) and $\sigma^{2}=10^{-4}$ (right).
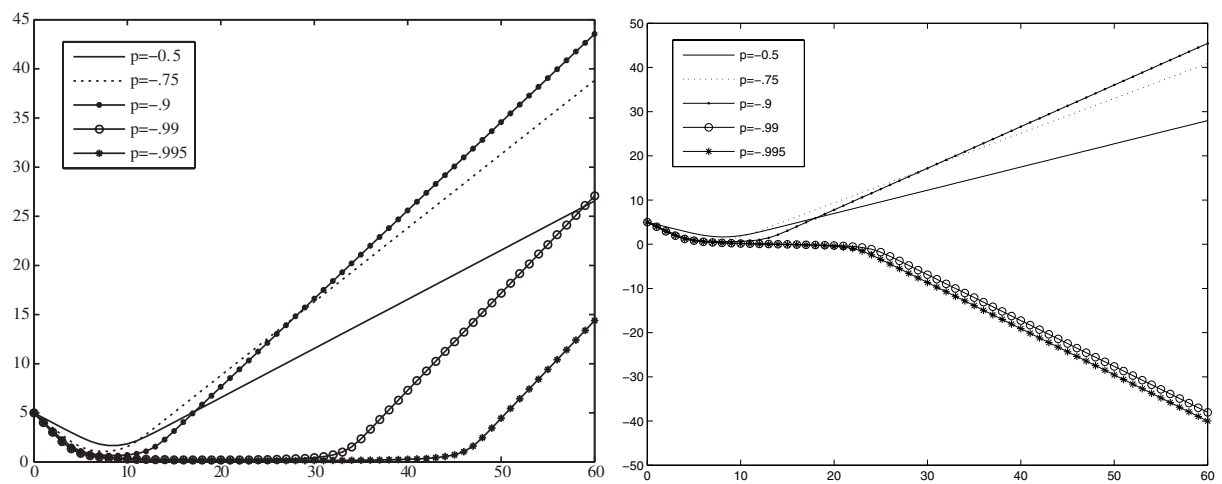

FIG. 2. One peakon catching up with another. The plots show the evolution of $q=q_{2}-q_{1}$ over time for several values of $p=p_{2}-p_{1}$, for $\sigma^{2}=0$ (left) and $\sigma^{2}=0.05$ (right).

metamorphoses [20, 27] can be obtained with the Lagrangian

$$
\ell(u, \nu)=\|u\|_{\mathfrak{g}}^{2}+\frac{1}{\sigma^{2}}\|\nu\|_{L^{2}}^{2} .
$$

If $w \in \mathfrak{g}$ and $n$ is an image, $w n=-\nabla n^{T} w$, so that $\left(\frac{\delta \ell}{\delta \nu} \diamond n \mid w\right)=\left(\frac{\delta \ell}{\delta \nu} \mid \nabla n^{T} w\right)$. Thus, since $\delta \ell / \delta \nu=2 \nu / \sigma^{2}$, the first equation is

$$
L_{\mathfrak{g}} u_{t}=-\frac{1}{\sigma^{2}} \nu_{t} \nabla n_{t}
$$



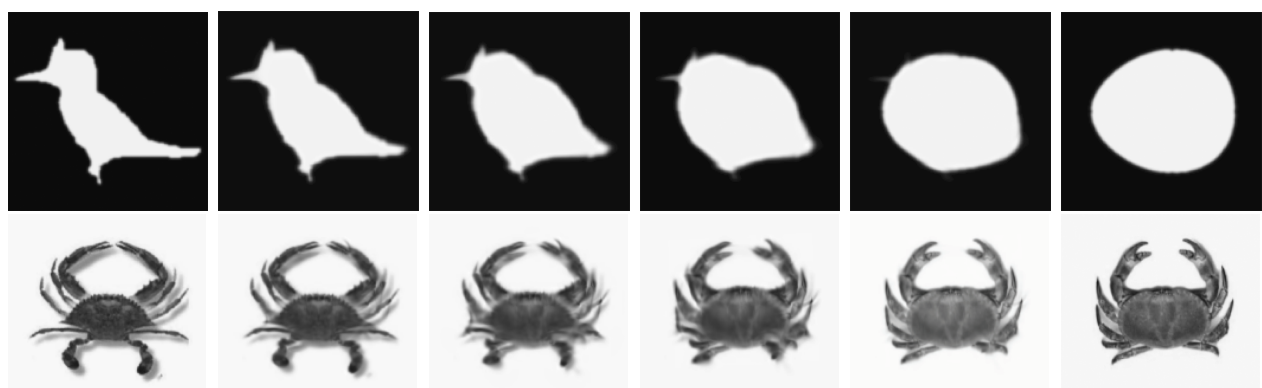

FIG. 3. Minimizing metamorphosis between images. The optimal trajectories for $n_{t}$ are computed between the first and last images in each row. The remaining images show $n_{t}$ at intermediate points in time.

Since $u \star(\delta \ell / \delta \nu)$ is defined by

$$
\begin{aligned}
\left(u \star\left(\frac{\delta \ell}{\delta \nu}\right) \mid \omega\right) & =\left(\frac{\delta \ell}{\delta \nu} \mid u \omega\right) \\
& =-\left(\frac{\delta \ell}{\delta \nu} \mid \nabla \omega^{T} u\right) \\
& =-\frac{1}{\sigma^{2}}\left(\nu \mid \nabla \omega^{T} u\right) \\
& =\frac{1}{\sigma^{2}}(\operatorname{div}(\nu u) \mid \omega),
\end{aligned}
$$

we obtain the second equation

$$
\dot{\nu}_{t}+\frac{1}{\sigma^{2}} \operatorname{div}\left(\nu_{t} u_{t}\right)=0 .
$$

As in the landmark case, denote $z=\nu / \sigma^{2}$ and rewrite the evolution equations in the form

$$
\left\{\begin{array}{l}
L_{\mathfrak{g}} u_{t}=-z_{t} \nabla n_{t}, \\
\dot{z}_{t}+\operatorname{div}\left(z_{t} u_{t}\right)=0, \\
\dot{n}_{t}+\nabla n_{t}^{T} u_{t}=\sigma^{2} z_{t} .
\end{array}\right.
$$

The existence and uniqueness of solutions for this system have been proved in [27]. From a visual point of view, image metamorphoses are similar to what is usually called "morphing" in computer graphics. The evolution of the image over time, $t \mapsto n_{t}$, is a combination of deformations and image intensity variation. Algorithms and experimental results for the solution of the boundary value problem (minimize the Lagrangian between two images) can be found in 20, 9. Some examples of minimizing geodesics are also provided in Figure 3

In 1D, letting $m=L_{\mathfrak{g}} u=\left(1-\partial_{x}^{2}\right) u$, the time evolving form of this system (as provided by (5), or by direct computation from (16)) becomes, with $\rho=\sigma z$ :

$$
\partial_{t} m+u \partial_{x} m+2 m \partial_{x} u=-\rho \partial_{x} \rho \quad \text { with } \quad \partial_{t} \rho+\partial_{x}(\rho u)=0 .
$$


This relates, with the important difference of a minus sign in front of $\rho \partial_{x} \rho$ in the first equation, to the two-component Camassa-Holm system studied in [6, 8, 17]. The system (13) in our case is equivalent to the compatibility for $d \lambda / d t=0$ of

$$
\begin{aligned}
& \partial_{x}^{2} \psi+\left(-\frac{1}{4}+m \lambda+\rho^{2} \lambda^{2}\right) \psi=0 \\
& \partial_{t} \psi=-\left(\frac{1}{2 \lambda}+u\right) \partial_{x} \psi+\frac{1}{2} \psi \partial_{x} u
\end{aligned}
$$

Image matching can also be seen under the semi-direct product point of view, since the action is linear and the Lagrangian takes the form (6) with $n^{-1} \nu=\nu$. This implies that the momentum, which is, in this case, the pair $\left(L_{\mathfrak{g}} u, z\right)$, is conserved in a fixed frame. Working out the conservation equation $\operatorname{Ad}_{(g, n)}^{*}\left(L_{\mathfrak{g}} u, z\right)=$ cst in this case yields the equations $L_{\mathfrak{g}} u_{t}+z_{t} \nabla n_{t}=$ cst and $z_{t}=\operatorname{det}\left(D g_{t}^{-1}\right) z_{0} \circ g_{t}^{-1}$. This last condition is the integrated form of the second equation in (12), while the first equation in (12) implies that in fact $L_{\mathfrak{g}} u_{t}+z_{t} \nabla n_{t}=0$, which is the horizontality condition in the quotient space $G$ (S) $N / G$.

6.3. Densities. We here let $N$ be a space of smooth functions $n: \Omega \rightarrow \mathbb{R}$ with the action $(g, n) \mapsto\left|\operatorname{det} D\left(g^{-1}\right)\right| n \circ g^{-1}$; i.e., $n$ deforms as a density. We consider the same Lagrangian as with images,

$$
\ell(u, \nu)=\frac{1}{2}\|u\|_{\mathfrak{g}}^{2}+\frac{1}{2 \sigma^{2}}\|\nu\|_{L^{2}}^{2} .
$$

For $w \in \mathfrak{g}$ and $n \in H$, we have $w n=-\nabla n^{T} w-n \operatorname{div}(w)=-\operatorname{div}(n w)$. This implies

$$
\begin{aligned}
\left(\frac{\delta \ell}{\delta \nu} \diamond n \mid w\right) & =-\frac{1}{\sigma^{2}}(\nu \mid \operatorname{div}(n w)) \\
& =\frac{1}{\sigma^{2}}(n \nabla \nu \mid w),
\end{aligned}
$$

yielding the first equation

$$
L_{\mathfrak{g}} u=\frac{1}{\sigma^{2}} n \nabla \nu
$$

Similarly, we get $u \star \nu=\nabla \nu^{T} u$ and the equation

$$
\dot{\nu}+\nabla \nu^{T} \nu=0 \text {. }
$$

This yields the system, where we have, as before, introduced $z=\nu / \sigma^{2}$ :

$$
\left\{\begin{array}{l}
L_{\mathfrak{g}} u=n \nabla z \\
\dot{z}+\nabla z^{T} u=0 \\
\dot{n}+\operatorname{div}(n u)=\sigma^{2} z
\end{array}\right.
$$

We are here also in the semi-direct product case, the equations for the conservation of momentum being $L_{\mathfrak{g}} u+n \nabla z=$ cst and $z=z_{0} \circ g^{-1}$. As for images, the constant in the first conservation equation vanishes for horizontal geodesics in $G$ (S) $N / G$. Optimal metamorphoses with densities are illustrated in Figure 4. 

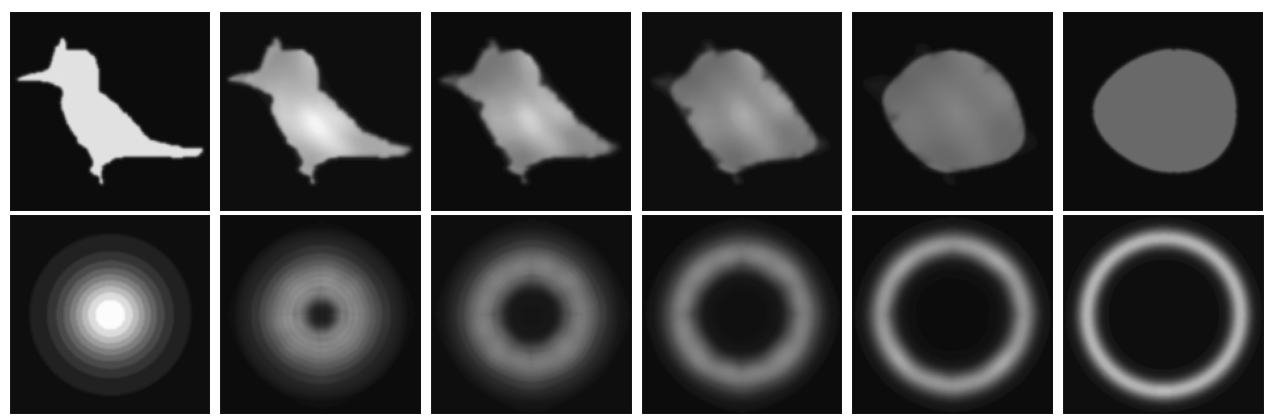

FIG. 4. Minimizing metamorphoses between densities with equal total mass. The optimal trajectories for $n_{t}$ are computed between the first and last densities in each row. The remaining images show $n_{t}$ at intermediate points in time.

6.4. Plane curves. We here consider matching unit-length curves $\gamma$ defined on the unit circle $S^{1}$, represented, as in 32, 21, 33, with their normalized tangent $\theta \mapsto \dot{\gamma}_{\theta}$ with $\left|\dot{\gamma}_{\theta}\right|=1 /(2 \pi)$. The set $N$ is therefore a set of functions $n: S^{1} \rightarrow S^{1}(1 /(2 \pi))$, where $S^{1}(r)$ is the sphere with radius $r$ in $\mathbb{R}^{2}$. We let $G$ be the group of diffeomorphisms of $S^{1}$ and consider the reduced Lagrangian

$$
\ell(u, \nu)=\int_{S^{1}} \dot{u}_{\theta}^{2} d \theta+\frac{1}{\sigma^{2}} \int_{S^{1}}|\nu|^{2} d \theta .
$$

We want to solve the metamorphosis problem while ensuring that curves are closed, which translates into:

$$
\int_{S}^{1} n_{t} d \theta=0
$$

To make (9) explicit, we need a local chart to compute the partial derivatives $\partial / \partial \nu$ and $\partial / \partial n$ ( $N$ is not a vector space here). Consider the representation $n=h_{\alpha}$ and $\nu=\sigma^{2} \rho h_{\alpha}^{\perp}$ with $h_{\alpha}=(\cos \alpha, \sin \alpha)$ and $h_{\alpha}^{\perp}=(-\sin \alpha, \cos \alpha)$. We then get the equations, with $\lambda_{t} \in \mathbb{R}^{2}$,

$$
\left\{\begin{array}{l}
-\frac{\partial^{2} u_{t}}{\partial \theta^{2}}+\rho_{t} \frac{\partial \alpha_{t}}{\partial \theta}=0, \\
\frac{\partial \rho_{t}}{\partial t}+\frac{\partial}{\partial \theta}\left(u_{t} \rho_{t}\right)=-\lambda_{t}^{T} h_{\alpha_{t}}^{\perp}, \\
\dot{\alpha}_{t}=\sigma^{2} \rho_{t}-u_{t} \frac{\partial \alpha_{t}}{\partial \theta}, \\
\int_{S^{1}} \dot{\alpha}_{t} h_{\alpha_{t}}^{\perp} d \theta=0 .
\end{array}\right.
$$

Interestingly, these equations can be notably simplified in the case $\sigma^{2}=1$, which has been considered in $\left[32,25,26,33\right.$. In this case, the change of variables $z_{t}^{2}=\dot{g}_{t} n_{t} \circ g_{t}$, where both sides are interpreted as complex numbers, reduces (17) to geodesic equations on a Grassmann manifold, on which explicit computations can be made 33. The case 

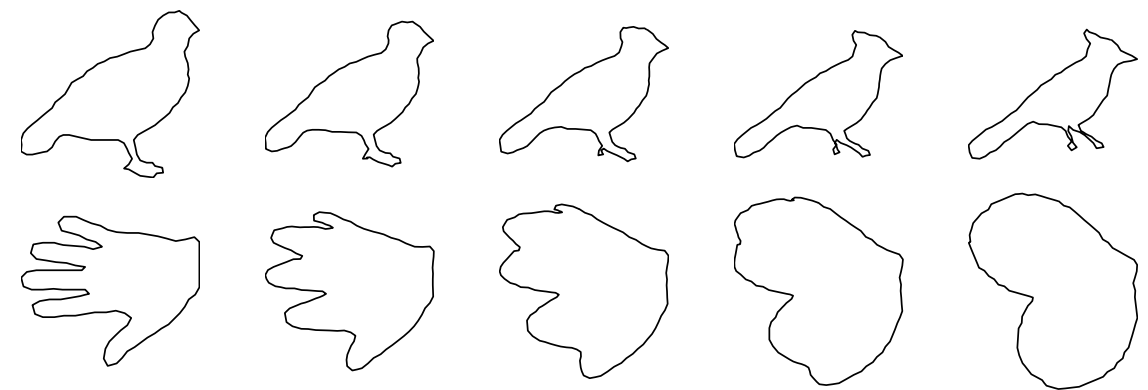

FIG. 5. Optimal metamorphoses between plane curves. The optimal trajectories for $n_{t}$ are computed between the first and last curves in each row. The remaining images show $n_{t}$ at intermediate points in time.

$\sigma^{2}=4$ is also interesting, and has been discussed in [15]. Figure 5 provides the result of curve metamorphosis using $\sigma^{2}=1$.

7. Measure metamorphoses. We now focus on extending the example in section 6.3 to also include singular measures. The $L^{2}$-norm that we have used between densities is therefore no longer available. We will rely on a construction that was introduced in [10.

We let $H$ be a reproducing kernel Hilbert space (RKHS) of functions over $\mathbb{R}^{d}$ and $N=$ $H^{*} ; H$ being an RKHS is equivalent to the fact that for all $x \in \mathbb{R}^{d}$, there exists a function $K_{x} \in H$ such that, for all $f \in H,\left\langle K_{x}, f\right\rangle_{H}=f(x)$. The kernel $K_{H}(x, y):=K_{x}(y)$ satisfies the equation $\left\langle K_{x}, K_{y}\right\rangle_{H}=K_{H}(x, y)$. It also provides an isometry between $N$ and $H$ via the relation $\eta \mapsto K_{H} \eta$ with $\left\langle K_{H} \eta, f\right\rangle_{H}=(\eta \mid f)$. This implies in particular that the dual inner product on $N$ is given by

$$
\langle\eta, \tilde{\eta}\rangle_{N}=\left(\eta \mid K_{H} \tilde{\eta}\right)
$$

Letting $G$ be as in the previous section, we want to study metamorphoses in $G \times N$. We define the action of $G$ on $N$ by (with $g \in G, \eta \in N$ and $f \in H$ )

$$
(g \eta \mid f)=(\eta \mid f \circ g) .
$$

This obviously generalizes the action on densities discussed in the previous section. For $\eta \in N$ and $w \in \mathfrak{g}$, we have, for all $f \in H,(w \eta \mid f)=\left(\eta \mid \nabla f^{T} w\right)$.

Since the action is linear, we can use the semi-direct product model and the Lagrangian

$$
\ell(u, \nu)=\frac{1}{2}\|u\|_{\mathfrak{g}}^{2}+\frac{1}{2 \sigma^{2}}\|\nu\|_{N}^{2} .
$$

To explicitly compute again (4) in this context, we need to compute $(\partial \ell / \partial \nu) \diamond n$. We let $f=\delta \ell / \delta \nu=\left(1 / \sigma^{2}\right) K_{H} \nu$. By definition, we have, for all $w \in \mathfrak{g}$,

$$
\begin{aligned}
(f \diamond n \mid w) & =-(f \mid w n) \\
& =-\left(n \mid \nabla f^{T} w\right) \\
& =-(\nabla f \otimes n \mid w) .
\end{aligned}
$$


We therefore obtain our first equation $(\delta \ell / \delta u)=\nabla f \otimes \nu$. Since $(f \mid u \eta)=\left(\eta \mid \nabla f^{T} u\right)$, the second equation, $\dot{f}_{t}+u_{t} \star f_{t}=0$, is the advection: $\dot{f}_{t}+\nabla^{T} f_{t} u_{t}=0$. This yields the system (with $L_{H}=K_{H}^{-1}$ )

$$
\left\{\begin{array}{l}
L_{\mathfrak{g}} u_{t}=\nabla f_{t} \otimes n_{t} \\
\dot{f}_{t}+\nabla f_{t}^{T} u_{t}=0 \\
\dot{n}_{t}-u_{t} n_{t}=\sigma^{2} L_{H} f_{t} .
\end{array}\right.
$$

From the second equation, we get $f_{t}=f_{0} \circ g_{t}^{-1}$. Using $g \dot{\eta}=\nu=\sigma^{2} L_{H} f$ and $g \eta=n$, we get

$$
n_{t}=g_{t} n_{0}+\sigma^{2} g_{t} \int_{0}^{t} g_{s}^{-1} K_{H}^{-1}\left(f_{0} \circ g_{s}^{-1}\right) d s .
$$

We therefore obtain integrated equations for measure metamorphoses

$$
\left\{\begin{array}{l}
L_{\mathfrak{g}} u_{t}=\nabla f_{t} \otimes n_{t} \\
n_{t}=g_{t} n_{0}+\sigma^{2} g_{t} \int_{0}^{t} g_{s}^{-1} L_{H}\left(f_{0} \circ g_{s}^{-1}\right) d s
\end{array}\right.
$$

with $\dot{g}_{t}=u_{t} \circ g_{t}$.

We now pass to the theoretical study of the existence of solutions for the initial value problem (IVP) and boundary value problem (BVP) for measure metamorphosis (with uniqueness in the IVP case). The next two sections are notably more technical than the rest of this paper. They are well isolated from it, however, and it is possible, if desired, to skip directly to section 10 .

\section{Existence of solutions for the measure metamorphosis IVP.}

8.1. Hypotheses on the Hilbert spaces. For the existence proofs to proceed, we need some conditions on the Hilbert spaces $\mathfrak{g}$ and $H$. They are essentially adapted to $H$ being equivalent to $H_{0}^{q}$ (the completion, in $H^{q}$, of $C^{\infty}$ functions with compact support), in which case $H^{-}=H_{0}^{q-1}$ and $H^{+}=H_{0}^{q+1}$ ( $q$ being large enough to ensure that $H^{q}$ is embedded in $C^{0}$ ).

In the following, cst represents a generic constant, and $C$ a generic continuous function of its parameters. We assume the existence of two spaces $H^{+}$and $H^{-}$and the following properties, valid for some $q \geq 1$.

(H1) If $\tilde{H}=H^{-}, H$ or $H^{+}$, we have: if $f \in \tilde{H}$ and $g \in C^{q}(\Omega)$, then $f \circ g \in \tilde{H}$ and

$$
\|f \circ g\|_{\tilde{H}} \leq C\left(\|g\|_{q, \infty}\right)\|f\|_{\tilde{H}} .
$$

(H2) For $f \in H^{+}$, and $g, \tilde{g}$ two $C^{q}$ diffeomorphisms,

$$
\|f \circ g-f \circ \tilde{g}\|_{H} \leq \operatorname{cst}\|f\|_{H^{+}} C\left(\max \left(\|g\|_{q, \infty},\|\tilde{g}\|_{q, \infty}\right)\right)\|g-\tilde{g}\|_{q, \infty} .
$$

(H3) For $f \in H$, define the operator $Q_{f}$ on $\mathfrak{g}$ by $Q_{f} w=\nabla f^{T} w$. Then for all $f \in H$ and $g \in C^{q}(\Omega), Q_{f}$ maps $\mathcal{X}^{q}(\Omega)$ to $H^{-}$with

$$
\left\|Q_{f} w\right\|_{H^{-}} \leq \operatorname{cst}\|f\|_{H}\|w\|_{q, \infty}
$$


for all $w \in \mathcal{X}^{q}(\Omega)$. (Here, $\mathcal{X}^{q}(\Omega)$ denotes the set of $C^{q}$ vector fields on $\Omega$ with the supremum norm of all derivatives of order less than q.)

(H4) If $f \in H^{+}$, then $K_{H}^{-1} f \in\left(H^{-}\right)^{*}$ and for all $z \in H^{-},\left(K_{H}^{-1} f \mid z\right) \leq \operatorname{cst}\|f\|_{H^{+}}\|z\|_{H^{-}}$.

$(\mathrm{H} 5) \mathfrak{g}$ is continuously included in $\mathcal{X}_{0}^{p}(\Omega)$ for $p>q+1$, where $\mathcal{X}_{0}^{p}$ is the completion of compactly supported vector fields in $\mathcal{X}^{p}(\Omega)$.

Denote $N^{+}=\left(H^{-}\right)^{*}$. We then have

Theorem 1. Under the hypotheses (H1) to (H3), for all $T>0$, there exists a unique solution to system (19) over $[0, T]$ with initial conditions $n_{0} \in N^{+}$and $f_{0} \in H^{+}$.

Proof. We prove existence for small enough $T$ with a fixed point argument. Consider the Hilbert space $L^{2}([0, T], \mathfrak{g})$, with norm

$$
\|u\|_{2, T}^{2}=\int_{0}^{T}\left\|u_{t}\right\|_{\mathfrak{g}}^{2} d t
$$

For $u \in L^{2}([0, T], \mathfrak{g})$, define $\Psi(u):=u^{\prime}$ given by

$$
\left\{\begin{array}{l}
u_{t}^{\prime}=K_{\mathfrak{g}}\left(\nabla f_{t} \otimes n_{t}\right) \\
n_{t}=g_{t} n_{0}+\sigma^{2} g_{t} \int_{0}^{t} g_{s}^{-1} L_{H}\left(f_{0} \circ g_{s}^{-1}\right) d s
\end{array}\right.
$$

with $\dot{g}_{t}=u_{t} \circ g_{t}$.

First note that the hypotheses imply that $\Psi$ is well defined and takes values in $L^{2}([0, T], \mathfrak{g})$. Indeed, by definition,

$$
\begin{aligned}
\left\langle u_{t}^{\prime}, w\right\rangle_{\mathfrak{g}} & =\left(\nabla f_{t} \otimes n_{t} \mid w\right) \\
& =\left(n_{t} \mid \nabla f_{t}^{T} w\right) \\
& \leq \operatorname{cst}\left\|n_{t}\right\|_{N}\left\|f_{t}\right\|_{H^{+}}\|w\|_{\tilde{q}, \infty} \\
& \leq \operatorname{cst}\left\|n_{t}\right\|_{N}\left\|f_{t}\right\|_{H^{+}}\|w\|_{\mathfrak{g}}
\end{aligned}
$$

so that a sufficient condition for $\nabla f_{t} \otimes n_{t} \in \mathfrak{g}^{*}$ is $n_{t} \in N$ and $f_{t} \in H^{+}$. Since $f_{t}=f_{0} \circ g_{t}^{-1}$, we have $\left\|f_{t}\right\|_{H^{+}} \leq \operatorname{cst}\left\|f_{0}\right\|_{H^{+}} C\left(\left\|g_{t}^{-1}\right\|_{q, \infty}\right)$. From [27, we have $\left\|g_{t}^{-1}\right\|_{q, \infty}=O\left(\|u\|_{2, T}\right)$, yielding

$$
\left\|f_{t}\right\|_{H^{+}} \leq \operatorname{cst}\left\|f_{0}\right\|_{H^{+}} C\left(\|u\|_{2, T}\right) .
$$

For $z \in H$, we have

$$
\begin{aligned}
\left(n_{t} \mid z\right) & =\left(n_{0} \mid z \circ g_{t}\right)+\sigma^{2} \int_{0}^{t}\left(K_{H}^{-1} f_{s} \mid z_{s} \circ g_{t} \circ g_{s}^{-1}\right) d s \\
& \leq\left(\left\|n_{0}\right\|_{N} C\left(\left\|g_{t}\right\|_{q, \infty}\right)+\sigma^{2} \int_{0}^{t}\left\|f_{s}\right\|_{H} C\left(\left\|g_{t} \circ g_{s}^{-1}\right\|_{q, \infty}\right) d s\right)\|z\|_{H}
\end{aligned}
$$

so that

$$
\left\|n_{t}\right\|_{N} \leq\left(\left\|n_{0}\right\|_{N}+\sigma^{2} t\left\|f_{0}\right\|_{H}\right) C\left(\|u\|_{2, T}\right)
$$

This implies that

$$
\left\|u_{t}^{\prime}\right\|_{\mathfrak{g}} \leq\left\|f_{0}\right\|_{H^{+}}\left(\left\|n_{0}\right\|_{N}+\sigma^{2} t\left\|f_{0}\right\|_{H}\right) C\left(\|u\|_{2, T}\right)
$$


and

$$
\left\|u^{\prime}\right\|_{2, T} \leq \operatorname{cst} \sqrt{T}\left\|f_{0}\right\|_{H^{+}}\left(\left\|n_{0}\right\|_{N}+\sigma^{2} T\left\|f_{0}\right\|_{H}\right) C\left(\|u\|_{2, T}\right) .
$$

In particular, this implies that, for any $M>0$, there exists a $T_{0}(M)$ (only depending on $\left\|n_{0}\right\|_{N^{+}}$and $\left.\left\|f_{0}\right\|_{H^{+}}\right)$such that, for $T<T_{0},\|u\|_{2, T} \leq M$ implies $\left\|u^{\prime}\right\|_{2, T} \leq M$. From now on, we assume that $T<T_{0}(M)$ with $M=1$.

Note that a similar computation also shows that $n_{t} \in N^{+}$: for $z \in H^{-}$, we have

$$
\begin{aligned}
\left(n_{t} \mid z\right) & =\left(n_{0} \mid z \circ g_{t}\right)+\sigma^{2} \int_{0}^{t}\left(K_{H}^{-1} f_{s} \mid z_{s} \circ g_{t} \circ g_{s}^{-1}\right) d s \\
& \leq\left(\left\|n_{0}\right\|_{N^{+}} C\left(\left\|g_{t}\right\|_{q, \infty}\right)+\sigma^{2} \int_{0}^{t}\left\|f_{s}\right\|_{H^{+}} C\left(\left\|g_{t} \circ g_{s}^{-1}\right\|_{q, \infty}\right) d s\right)\|z\|_{H^{-}}
\end{aligned}
$$

so that

$$
\left\|n_{t}\right\|_{N^{+}} \leq\left(\left\|n_{0}\right\|_{N^{+}}+\sigma^{2} t\left\|f_{0}\right\|_{H^{+}}\right) C\left(\|u\|_{2, T}\right) .
$$

We now ensure that $\Psi$ is contractive. Take $u, \tilde{u}$ with $\max \left(\|u\|_{2, T},\|\tilde{u}\|_{2, T}\right) \leq 1$. We want to show that $T$ can be chosen so that $\left\|u^{\prime}-\tilde{u}^{\prime}\right\|_{2, T} \leq \rho\|u-\tilde{u}\|_{2, T}$ with $\rho<1$, where $u^{\prime}=\Psi(u)$ and $\tilde{u}^{\prime}=\Psi(\tilde{u})$. We have

$$
\begin{aligned}
\left\|u_{t}^{\prime}-\tilde{u}_{t}^{\prime}\right\|_{\mathfrak{g}} & =\left\|\nabla f_{t} \otimes n_{t}-\nabla \tilde{f}_{t} \otimes \tilde{n}_{t}\right\|_{\mathfrak{g}^{*}} \\
& \leq\left\|\nabla\left(f_{t}-\tilde{f}_{t}\right) \otimes n_{t}\right\|_{\mathfrak{g}^{*}}+\left\|\nabla \tilde{f}_{t} \otimes\left(n_{t}-\tilde{n}_{t}\right)\right\|_{\mathfrak{g}^{*}} \\
& \leq \operatorname{cst}\left\|n_{t}\right\|_{N^{+}}\left\|f_{t}-\tilde{f}_{t}\right\|_{H}+\operatorname{cst}\left\|n_{t}-\tilde{n}_{t}\right\|_{N}\left\|\tilde{f}_{t}\right\|_{H^{+}} .
\end{aligned}
$$

(Here, we have used the fact that for $n \in N^{+}$and $z \in H$, we have both $(n \mid z) \leq$ $\|n\|_{N}\|z\|_{H}$ and $(n \mid z) \leq\|n\|_{N^{+}}\|z\|_{H^{-}}$.)

Upper bounds for $\left\|\tilde{f}_{t}\right\|_{H^{+}},\left\|n_{t}\right\|_{N}$ and $\left\|n_{t}\right\|_{N^{+}}$are provided by equations (21), (22) and (23).

Moreover, from (H2), we have

$$
\left\|f_{t}-\tilde{f}_{t}\right\|_{H} \leq C\left(\max \left(\left\|g_{t}^{-1}\right\|_{q, \infty},\left\|\tilde{g}_{t}^{-1}\right\|_{q, \infty}\right)\left\|f_{0}\right\|_{H^{+}}\left\|g_{t}^{-1}-\tilde{g}_{t}^{-1}\right\|_{q, \infty}\right.
$$

and, using [27], we have $\left\|g_{t}^{-1}-\tilde{g}_{t}^{-1}\right\|_{q+1, \infty} \leq \operatorname{cst}\|u-\tilde{u}\|_{2, T}$ so that

$$
\left\|n_{t}\right\|_{N^{+}}\left\|f_{t}-\tilde{f}_{t}\right\|_{H} \leq \operatorname{cst}\left(\left\|n_{0}\right\|_{N^{+}}+\sigma^{2} t\left\|f_{0}\right\|_{H^{+}}\right)\left\|f_{0}\right\|_{H^{+}}\|u-\tilde{u}\|_{2, T} .
$$

For $z \in H$, we can write

$$
\begin{aligned}
\left(n_{t}-\tilde{n}_{t} \mid z\right)= & \left(n_{0} \mid z \circ g_{t}-z \circ \tilde{g}_{t}\right) \\
& +\sigma^{2} \int_{0}^{t}\left(K_{H}^{-1} f_{s} \mid z \circ g_{t} \circ g_{s}^{-1}-z \circ \tilde{g}_{t} \circ \tilde{g}_{s}^{-1}\right) d s \\
& +\sigma^{2} \int_{0}^{t}\left(K_{H}^{-1}\left(f_{s}-\tilde{f}_{s}\right) \mid z \circ \tilde{g}_{t} \circ \tilde{g}_{s}^{-1}\right) d s \\
(\mathrm{i}) \leq \quad & \operatorname{cst}\left\|n_{0}\right\|_{N^{+}}\|z\|_{H}\left\|g_{t}-\tilde{g}_{t}\right\|_{q, \infty} \\
& +\operatorname{cst} \sigma^{2} t\left\|f_{0}\right\|_{H^{+}}\|z\|_{H} \max _{s}\left\|g_{t} \circ g_{s}^{-1}-\tilde{g}_{t} \circ \tilde{g}_{s}^{-1}\right\|_{q, \infty} \\
(\mathrm{ii}) \quad & +\operatorname{cst} \sigma^{2} t\left\|f_{0}\right\|_{H^{+}}\|z\|_{H} \max _{s}\left\|g_{s}-\tilde{g}_{s}\right\|_{q, \infty}\left\|\tilde{g}_{t} \circ \tilde{g}_{s}^{-1}\right\|_{q, \infty} .
\end{aligned}
$$

For (i), we have used

$$
\left(n_{0} \mid z \circ g_{t}-z \circ \tilde{g}_{t}\right) \leq\left\|n_{0}\right\|_{N^{+}}\left\|z \circ g_{t}-z \circ \tilde{g}_{t}\right\|_{H^{-}} \leq \operatorname{cst}\left\|n_{0}\right\|_{N^{+}}\|z\|_{H}\left\|g_{t}-\tilde{g}_{t}\right\|_{p, \infty} .
$$


For (ii), we have used the same argument combined with the fact that, since $f_{s} \in H^{+}$, $K_{H}^{-1} f_{s} \in N^{+}$with $\left\|K_{H}^{-1} f_{s}\right\|_{N^{+}} \leq \operatorname{cst}\left\|f_{s}\right\|_{H^{+}}$. For (iii), the computation uses the fact that for $\tilde{z} \in H$,

$$
\left(K_{H}^{-1}\left(f_{s}-\tilde{f}_{s}\right) \mid \tilde{z}\right) \leq\left\|f_{s}-\tilde{f}_{s}\right\|_{H}\|z\|_{H} .
$$

We therefore obtain the inequality

$$
\left\|n_{t}-\tilde{n}_{t}\right\|_{H} \leq \operatorname{cst}\left(\left\|n_{0}\right\|_{N^{+}}+\left\|f_{0}\right\|_{H^{+}}\right)\|u-\tilde{u}\|_{2, T} .
$$

Collecting the previous estimates, we have

$$
\left\|u_{t}^{\prime}-\tilde{u}_{t}^{\prime}\right\|_{\mathfrak{g}} \leq F\left(\left\|n_{0}\right\|_{N^{+}},\left\|f_{0}\right\|_{H^{+}}\right)\|u-\tilde{u}\|_{2, T},
$$

where $F$ is a polynomial. This implies

$$
\left\|u^{\prime}-\tilde{u}^{\prime}\right\|_{2, T} \leq \sqrt{T} F\left(\left\|n_{0}\right\|_{N^{+}},\left\|f_{0}\right\|_{H^{+}}\right)\|u-\tilde{u}\|_{2, T},
$$

so that $\Psi$ is contractive for small enough $T$.

The extension from small times to all times can be done as in [27], and we only sketch the details. According to the small time computation, the length over which the solution exists is at least the inverse of a polynomial function of $\left\|n_{0}\right\|_{N^{+}}$and $\left\|f_{0}\right\|_{H^{+}}$. One will therefore be able to extend this equation beyond $T$, unless either $\left\|n_{t}\right\|_{N^{+}}$or $\left\|f_{t}\right\|_{H^{+}}$tends to infinity when $t$ tends to $T$. From (21) and (22), this can happen only if $\|u\|_{2, t}$ tends to infinity when $t$ tends to $T$. But this is impossible, because (19) is a geodesic equation on $G$ (S) $N$ which implies that the value of $h_{t}:=\left\|u_{t}\right\|_{\mathfrak{g}}^{2}+\left(1 / \sigma^{2}\right)\left\|\nu_{t}\right\|_{N}^{2}$ is constant over time. This implies in particular that $\|u\|_{2, t}^{2} \leq t h_{0}$ and therefore cannot tend to infinity in finite time.

9. Existence of solutions for the measure metamorphosis BVP. Our goal in this section is to prove that, under some conditions on $H$, the boundary value problem for measure metamorphoses (BVP) always has solutions. This problem requires us to minimize, with fixed $n_{0}$ and $n_{1}$,

$$
E(u, n):=\int_{0}^{1}\left\|u_{t}\right\|_{\mathfrak{g}}^{2} d t+\frac{1}{\sigma^{2}} \int_{0}^{1}\left\|\dot{n}_{t}-u_{t} n_{t}\right\|_{N}^{2} d t .
$$

Letting $f_{t}=K_{H}\left(\dot{n}_{t}-u_{t} n_{t}\right)$, the problem is equivalent to minimizing

$$
E(u, f):=\int_{0}^{1}\left\|u_{t}\right\|_{\mathfrak{g}}^{2} d t+\sigma^{2} \int_{0}^{1}\left\|f_{t}\right\|_{H}^{2} d t
$$

with boundary

$$
n_{1}=g_{1} n_{0}+\sigma^{2} g_{1} \int_{0}^{1} g_{s}^{-1}\left(K_{H}^{-1} f_{s}\right) d s
$$

We have

Theorem 2. Assume that (H1) and (H2) hold and that $H^{+}$is dense in $H$. Then, for any given $n_{0}, n_{1} \in H$, there exists a minimizer for the BVP. 
Proof. Consider a minimizing sequence $\left(u^{(k)}, f^{(k)}\right)$. Using a time change if necessary, we can ensure that $c_{k}:=\left\|u_{t}^{(k)}\right\|_{\mathfrak{g}}^{2}+\sigma^{2}\left\|f_{t}^{(k)}\right\|^{2}$ is independent of time and therefore bounded. Moreover, we can extract a subsequence (still denoted $\left.\left(u^{(k)}, f^{(k)}\right)\right)$ which weakly converges to some $(u, f)$ in $L^{2}([0,1], \mathfrak{g} \times H)$, equipped with the norm $\left\|\left(u^{\prime}, f^{\prime}\right)\right\|=$ $E\left(u^{\prime}, f^{\prime}\right)$. This implies that $E(u, f) \leq \liminf E\left(u^{(k)}, f^{(k)}\right)$ so that the only thing that needs to be shown is that the boundary condition is still satisfied; namely, letting

$$
n_{1}^{\prime}=g_{1} n_{0}+\sigma^{2} g_{1} \int_{0}^{1} g_{s}^{-1}\left(K_{H}^{-1} f_{s}\right) d s
$$

with $\dot{g}_{t}=u_{t} \circ g_{t}$, we have $n_{1}^{\prime}=n_{1}$. For this, it suffices to show that, for $z$ in a dense subset of $H$, we have $\left(n_{1}^{\prime} \mid z\right)=\left(n_{1} \mid z\right)$. Since, for all $k$, we have

$$
n_{1}=g_{1}^{(k)} n_{0}+\sigma^{2} g_{1}^{(k)} \int_{0}^{1}\left(g_{s}^{(k)}\right)^{-1}\left(K_{H}^{-1} f_{s}^{(k)}\right) d s,
$$

it suffices to show that, for $z$ in a dense subset of $H$, we have

$$
\left(n_{0} \mid z \circ g_{1}^{(k)}\right) \rightarrow\left(n_{0} \mid z \circ g_{1}\right)
$$

and

$$
\int_{0}^{1}\left(K_{H}^{-1} f_{s}^{(k)} \mid z \circ g_{1}^{(k)} \circ\left(g_{s}^{(k)}\right)^{-1}\right) d s \rightarrow \int_{0}^{1}\left(K_{H}^{-1} f_{s} \mid z \circ g_{1} \circ g_{s}^{-1}\right) d s .
$$

Because of the weak convergence of $u^{(n)}$ to $u$, the flows $g^{(n)}$ converge to $g$ for the $(p-1, \infty)$-norm, uniformly in time 27 . Taking $z \in H^{+}$, we have

$$
\left\langle n_{0}, z \circ g_{1}^{(k)}-z \circ g_{1}\right\rangle \leq C\left(\sup _{t}\left(\left\|g_{t}^{(k)}\right\|_{p, \infty},\left\|g_{t}\right\|_{p, \infty}\right)\right)\left\|n_{0}\right\|_{H^{*}}\|z\|_{H^{+}} \sup _{t}\left\|g_{t}^{(k)}-g_{t}\right\|_{p, \infty},
$$

which tends to 0 so that (26) is true for all $z \in H^{+}$. Splitting the terms in (27), it suffices to show that for all $s \in[0,1]$,

$$
\int_{0}^{1}\left(K_{H}^{-1} f_{s}^{(k)} \mid z \circ g_{1}^{(k)} \circ\left(g_{s}^{(k)}\right)^{-1}\right) d s-\int_{0}^{1}\left(K_{H}^{-1} f_{s}^{(k)} \mid z \circ g_{1} \circ g_{s}^{-1}\right) \rightarrow 0
$$

and

$$
\int_{0}^{1}\left(K_{H}^{-1} f_{s}^{(k)} \mid z \circ g_{1} \circ\left(g_{s}\right)^{-1}\right) d s-\int_{0}^{1}\left(K_{H}^{-1} f_{s} \mid z \circ g_{1} \circ g_{s}^{-1}\right) d s \rightarrow 0 .
$$

For (28), and because we have ensured that $\left\|f_{s}^{(k)}\right\|_{H}$ and $\left\|u_{s}^{(k)}\right\|_{\mathfrak{g}}$ are uniformly bounded, it suffices to show that each term in the integral tends to 0 and then use the dominated convergence theorem. The left-hand term of (28) is bounded in absolute value by

$$
\operatorname{cst}\left\|f_{s}^{(k)}\right\|_{H}\|z\|_{H^{+}}\left\|g_{1}^{(k)} \circ\left(g_{s}^{(k)}\right)^{-1}-g_{1} \circ g_{s}^{-1}\right\|_{q, \infty},
$$

which tends to 0 , so that (28) holds. To prove (29), we only need the fact that $z \circ g_{1} \circ g_{s}^{-1}$ belongs to $H$ and the weak convergence of $f^{(k)}$ to $f$.

This shows that $n_{1}^{\prime}=n_{1}$ and concludes the proof of the theorem. 
9.1. Remark. Equations (19) have been obtained from general formulae that were derived under the assumption that $G$ is a Lie group (which is not the case here). It is important to rigorously recompute the Euler equation to reconnect the IVP and the BVP. The variation with respect to $u$ is straightforward and provides the first equation in (19).

We now discuss the minimization in $n$ with fixed $u$. Letting $\eta=g^{-1} n$ the problem with fixed $u$ (and therefore fixed $g$ ) is equivalent to the minimization of

$$
F(\eta)=\int_{0}^{1}\left\|g_{t} \dot{\eta}_{t}\right\|_{N}^{2} d t
$$

with fixed boundary conditions $\eta_{0}=n_{0}$ and $\eta_{1}=g_{1}^{-1} n_{1}$.

Assume the following hypothesis:

(H1b) $z \rightarrow z \circ g$ is weakly continuous for $z \in H\left(\right.$ where $\left.H^{*}=N\right)$.

For $g \in G$, introduce the operator $K_{H}^{g}$ defined by $K_{H}^{g} \eta=K_{H}(g \eta) \circ g$. With this notation,

$$
F(\eta)=\int_{0}^{1}\left(\dot{\eta}_{t} \mid K_{H}^{g_{t}} \dot{\eta}_{t}\right)_{N} d t
$$

Let $L_{H}^{g}=\left(K_{H}^{g}\right)^{-1}$, i.e., $L_{H}^{g} f=g^{-1} . L_{H}\left(f \circ g^{-1}\right)$, and denote

$$
\bar{L}_{H}^{g}=\int_{0}^{1} L_{H}^{g_{t}} d t
$$

We have for any $f \in H$,

$$
\left(\bar{L}_{H}^{g} f \mid f\right)=\int_{0}^{1}\left(L_{H}^{g_{t}} f \mid f\right) d t=\int_{0}^{1}\left\|f \circ g_{t}^{-1}\right\|_{H}^{2} d t \geq C\|f\|_{H}^{2},
$$

where the last inequality comes from (H1) with $C=\left[\sup _{t} C\left(\left\|g_{t}^{-1}\right\|_{q, \infty}\right)\right]^{-1}$. Thus, $\bar{L}_{H}^{g} f=$ 0 if and only if $f=0$ and $\bar{L}_{H}^{g}(H)$ is dense in $H^{*}$. If we prove that $\bar{L}_{H}^{g}(H)$ is closed, we will get that $\bar{L}_{H}^{g}(H): H \rightarrow H^{*}$ is invertible. Let $\left(f_{n}\right)_{n \geq 0}$ be a sequence in $H$ and $\eta \in H^{*}$ such that $\bar{L}_{H}^{g}\left(f_{n}\right) \rightarrow \eta$ in $H^{*}$. Then we get from (31) that $f_{n}$ is bounded in $H$ and we can assume that it admits a weak limit $f_{\infty}$ in $H$. Thus $\left(\bar{L}_{H}^{g} f_{\infty} \mid f\right)=$ $\int_{0}^{1}\left\langle f_{\infty} \circ g_{t}^{-1}, f \circ g_{t}^{-1}\right\rangle_{H} d t=\lim \int_{0}^{1}\left\langle f_{n} \circ g_{t}^{-1}, f \circ g_{t}^{-1}\right\rangle_{H} d t$, where the last equality comes from $(\mathrm{H} 1 \mathrm{~b})$ and the dominated convergence theorem. This yields $\left(\bar{L}_{H}^{g} f_{\infty} \mid f\right)=(\eta \mid f)$ for any $f \in H$ so that $\eta=\bar{L}_{H}^{g} f_{\infty}$ and $\bar{L}_{H}^{g}(H)$ is closed.

Let $f_{0}$ be such that $\bar{L}_{H}^{g} f_{0}=\eta_{1}-\eta_{0}$ and define

$$
\tilde{\eta}_{t}=\eta_{0}+\int_{0}^{t} L_{H}^{g_{s}} f_{0} d s
$$

for any $t \in[0,1]$. We now prove that, for any $\left(\dot{\eta}_{t}\right) \in L^{2}\left([0,1], H^{*}\right)$ with $\eta_{1}$ and $\eta_{0}$ fixed,

$$
F(\eta-\tilde{\eta})=F(\eta)+\text { cst. }
$$

We have

$$
F(\eta-\tilde{\eta})=F(\eta)+F(\tilde{\eta})-2 \int_{0}^{1}\left(\dot{\eta}_{t} \mid K_{H}^{g_{t}} \dot{\tilde{\eta}}_{t}\right) d t
$$

and $\int_{0}^{1}\left(\dot{\eta}_{t} \mid K_{H}^{g_{t}} \dot{\tilde{\eta}}_{t}\right) d t=\int_{0}^{1}\left(\dot{\eta}_{t} \mid f_{0}\right) d t=\left(\eta_{1}-\eta_{0} \mid f_{0}\right)$, so that the result is proved. 
We therefore have proved that minimizing $E$ with respect to $u, n$ is the same as minimizing

$$
\tilde{E}(u)=\int_{0}^{1}\left\|u_{t}\right\|_{\mathfrak{g}}^{2} d t+\frac{1}{\sigma^{2}}\left(\eta_{1}-\eta_{0} \mid\left(\bar{L}_{H}^{g}\right)^{-1}\left(\eta_{1}-\eta_{0}\right)\right)
$$

with respect to $u$. We also have the expression (32) for the optimal $\eta$ which is consistent with the second equation in (19).

10. A computational ansatz for point measure matching. The previous theorems provide a rigorous foundation for the measure matching approach being considered. However, an important issue needs to be addressed. The space $N$, which has been introduced in order to take advantage of its Hilbert structure, is a big space that contains distributions that are more singular than measures. Now, when matching two measures $n_{0}$ and $n_{1}$, the question naturally arises of whether the optimal evolution, i.e., the measure $n_{t}$, can turn up being more singular than measures, since the existence theorem only ensures that it belongs to $N$.

The second equation in (19) indicates that this should not be the case, since it says that

$$
n_{t}=g_{t} n_{0}+g_{t} \int_{0}^{t} L_{H}^{g_{s}}\left(\bar{L}_{H}^{g}\right)^{-1}\left(g_{1}^{-1} n_{1}-n_{0}\right) d s
$$

where $L_{H}^{g}$ and $\bar{L}_{H}^{g}$ are defined above and in equation (30). Thinking of $L_{H}$ as a differential operator, the number of derivatives computed by $L_{H}^{g_{s}}$ is "canceled" by the pre-application of $\left(\bar{L}_{H}^{g}\right)^{-1}$ so that $n_{t}$ should not be more singular than $\left(g_{1}^{-1} n_{1}-n_{0}\right)$, which is a measure. It is therefore reasonable to conjecture that when $n_{0}$ and $n_{1}$ are weighted sums of Dirac measures (which is a case of practical interest), then $n_{t}$ is a measure which has an absolutely continuous part, and a singular part which is also a sum of Dirac measures. More precisely, if

$$
n_{0}=\sum_{k=1}^{q} \alpha_{k}^{(0)} \delta_{x_{k}^{(0)}}, \quad n_{1}=\sum_{k=1}^{r} \beta_{k}^{(1)} \delta_{y_{k}^{(1)}},
$$

a reasonable ansatz for $n_{t}$ is

$$
n_{t}=\sum_{k=1}^{r} \alpha_{k}(t) \delta_{x_{k}(t)}+\sum_{k=1}^{r} \beta_{k}(t) \delta_{y_{k}(t)}+f(t, .) d x .
$$

Assuming this, the Lagrangian $\ell\left(v_{t}, n_{t}, \nu_{t}\right)$ can be considered as a function of $\left(\alpha_{k}(t)\right)$, $\left(\beta_{k}(t)\right),\left(x_{k}(t)\right),\left(y_{k}(t)\right), f(t,$.$) and their time derivatives, with an explicit expression in$ terms of the kernel $K_{H}$ and its space derivatives that we do not provide here, since it is quite lengthy. Minimization can then be done with standard methods, with boundary conditions $\alpha_{k}(0)=\alpha_{k}^{(0)}, \beta_{k}(0)=0, \alpha_{k}(1)=0, \beta_{k}(1)=\beta_{k}^{(1)}, x_{k}(0)=x_{k}^{(0)}, y_{k}(1)=y_{k}^{(1)}$ and $f(0,)=.f(1,)=$.0 .

11. More metamorphosis. Without getting into the level of rigor and detail developed with measure metamorphosis, we now review additional situations in which metamorphoses can be used. At the difference of the examples considered in section 6 , the following models have not yet been solved numerically, nor has a theoretical analysis 
been developed although we expect that the previous proofs of existence of solutions can be modified to work in these cases also.

11.1. Singular image metamorphosis. In section 7 , we have extended density metamorphosis to a context that includes singular measures. A similar analysis can be made to extend image metamorphosis to generalized functions. Let $H$ be a space of smooth scalar functions and $N=H^{*}$ as before. The extension to $N$ of the action of diffeomorphisms on images is $(g, n) \mapsto g n$ with

$$
(g n \mid f)=(n \mid \operatorname{det}(D g) f \circ g),
$$

the infinitesimal action being $(u n \mid f)=(n \mid \operatorname{div}(f u))$. Take as before the simplest reduced Lagrangian

$$
\ell(u, \nu)=\|u\|_{\mathfrak{g}}^{2}+\frac{1}{\sigma^{2}}\|\nu\|_{N}^{2} .
$$

We have, letting $f=\left(1 / \sigma^{2}\right) K_{H} \nu$,

$$
\left(\frac{\delta \ell}{\delta \nu} \diamond n \mid u\right)=(f \nabla n \mid u)
$$

and

$$
\left(u \star \frac{\delta \ell}{\delta \nu} \mid \omega\right)=(\omega \mid \operatorname{div}(f u))
$$

with the notation $(\nabla n \mid w)=-(n \mid \operatorname{div} w)$. We therefore obtain the generalized version of (12):

$$
\left\{\begin{array}{l}
L_{\mathfrak{g}} u_{t}=-f_{t} \nabla n_{t}, \\
\dot{f}_{t}+\operatorname{div}\left(f_{t} u_{t}\right)=0, \\
\dot{n}_{t}-u_{t} n_{t}=\sigma^{2} L_{H} f_{t} .
\end{array}\right.
$$

As for measures, this leads to the integrated equations

$$
\left\{\begin{array}{l}
L_{\mathfrak{g}} u_{t}=-f_{t} \nabla n_{t}, \\
n_{t}=g_{t} n_{0}+\sigma^{2} g_{t} \int_{0}^{t} g_{s}^{-1} L_{H}\left(f_{0} \circ g_{s}^{-1} \operatorname{det}\left(g_{s}^{-1}\right)\right) d s
\end{array}\right.
$$

with $\dot{g}_{t}=u_{t} \circ g_{t}$.

In the $1 \mathrm{D}$ case, with $\rho=\sigma f$ and $m=L_{\mathfrak{g}} u=\left(1-\partial_{x}^{2}\right) u$, we get a new version of (13):

$$
\partial_{t} m+u \partial_{x} m+2 m \partial_{x} u=-\rho \partial_{x} L_{H} \rho \quad \text { with } \quad \partial_{t} \rho+\partial_{x}(\rho u)=0 .
$$

11.2. Smooth image metamorphosis. We can go in the opposite direction and consider norms that will apply to smooth images in the metamorphosis formulation. Namely, keeping the notation of the previous section, we can define

$$
\ell(u, \nu)=\|u\|_{\mathfrak{g}}^{2}+\frac{1}{\sigma^{2}}\|\nu\|_{H}^{2} .
$$


Since the situation is completely symmetrical, we can immediately write the new system, with $f=\left(1 / \sigma^{2}\right) L_{H} \nu$, as

$$
\left\{\begin{array}{l}
L_{\mathfrak{g}} u_{t}=-f_{t} \nabla n_{t}, \\
\dot{f}_{t}+\operatorname{div}\left(f_{t} u_{t}\right)=0, \\
\dot{n}_{t}-u_{t} n_{t}=\sigma^{2} K_{H} f_{t} .
\end{array}\right.
$$

The second equation must be understood in a generalized sense, $f$ being advected by the flow as a measure.

An interesting feature of this system is that it admits singular solutions for the pair $\left(L_{\mathfrak{g}} u, f\right)$. Indeed, assume that $f_{0}=\sum_{k=1}^{Q} w^{(k)} \delta_{x_{0}^{(k)}}$ is a sum of weighted point masses. The second equation in (36) implies that

$$
f_{t}=\sum_{k=1}^{Q} w^{(k)} \delta_{x_{t}^{(k)}}
$$

with $\dot{x}_{t}^{(k)}=u_{t}\left(x_{t}^{(k)}\right)$. We also have

$$
L_{\mathfrak{g}} u_{t}=\sum_{k=1}^{Q} a_{t}^{(k)} \otimes \delta_{x_{t}^{(k)}}
$$

with $a_{t}^{(k)}=w^{(k)} \nabla n\left(x_{t}^{(k)}\right)$ and $n$ evolves according to the last equation in the system.

Exploiting this very simple structure can lead to interesting new methods for the analysis of smooth (or smoothed) images and will be considered in future work. The 1D evolution equation associated to this context is (letting, again, $\rho=\sigma f$ )

$$
\partial_{t} m+u \partial_{x} m+2 m \partial_{x} u=-\rho \partial_{x} K_{H} \rho \quad \text { with } \quad \partial_{t} \rho+\partial_{x}(\rho u)=0 .
$$

11.3. Smooth densities. Obviously, the same reduced Lagrangian can be used with densities. Using $(\nabla f \mid u)=-(f \mid \operatorname{div}(u))$ as a definition of $\nabla f$ for generalized functions, we get the system:

$$
\left\{\begin{array}{l}
L_{\mathfrak{g}} u_{t}=-\nabla n_{t} f_{t}-n_{t} \nabla f_{t}, \\
\dot{f}_{t}+u_{t}^{T} \nabla f_{t}=0, \\
\dot{n}_{t}+\operatorname{div}\left(u_{t} n_{t}\right)=\sigma^{2} K_{H} f_{t} .
\end{array}\right.
$$

Here also, singular solutions in $\left(L_{\mathfrak{g}} u, f\right)$ exist, although (as seen from the first equation), $L_{\mathfrak{g}} u$ is now one derivative less regular than $f$.

12. Conclusion. We have provided a general framework for the pattern matching theory of metamorphoses. The equations provided are quite versatile and adapted to any context in which a Lie group acts on a manifold.

In the particular case of diffeomorphisms acting on generalized functions, we have obtained a new set of equations, and showed that they had solutions, and so does the initial variational problem. Our equations seem to indicate that, when matching measures, 
metamorphoses do not generate additional singularities, but that they may introduce smooth components that did not appear in the initial problem.

Open for future work is the interesting problem of building an efficient numerical implementation of measure metamorphoses, and their use in specific pattern matching applications.

\section{REFERENCES}

[1] V. I. Arnold. Sur un principe variationnel pour les écoulements stationnaires des liquides parfaits et ses applications aux problèmes de stabilité non linéaires. J. Mécanique, 5:29-43, 1966.

[2] R. Bajcsy and C. Broit. Matching of deformed images. In The 6th international conference in pattern recognition, pages 351-353, 1982.

[3] M. F. Beg, M. I. Miller, A. Trouvé, and L. Younes. Computing large deformation metric mappings via geodesic flows of diffeomorphisms. Int. J. Comp. Vis., 61(2):139-157, 2005.

[4] F. L. Bookstein, Principal warps: Thin plate splines and the decomposition of deformations. IEEE Trans. PAMI, 11(6):567-585, 1989.

[5] V. Camion and L. Younes. Geodesic interpolating splines. In M. Figueiredo, J. Zerubia, and A. K. Jain, editors, EMMCVPR 2001, volume 2134 of Lecture notes in computer sciences. Springer, 2001.

[6] M. Chen, S. Liu, and Y. Zhang. A two-component generalization of the Camassa-Holm equation and its solutions. Lett. Math. Phys., 75:1-15, 2005. MR.2207043 (2006k:37177)

[7] P. Dupuis, U. Grenander, and M. Miller. Variational problems on flows of diffeomorphisms for image matching. Quarterly of Applied Math. 56:587-600, 1998. MR1632326 (99f:49006)

[8] G. Falqui. On a Camassa-Holm type equation with two dependent variables. J. Phys. A: Math. Gen., 39:327-342, 2006. MR2198963 (2006k:37179)

[9] L. Garcin and L. Younes. Geodesic image matching: A wavelet based energy minimization scheme. In Proceedings of EMMCVPR 2005, volume 3757 of Lecture Notes in Computer Science, pages 349-364, 2005.

[10] J. Glaunès, A. Trouvé, and L. Younes. Diffeomorphic matching of distributions: A new approach for unlabelled point-sets and sub-manifolds matching. In Proceedings of CVPR'04, 2004.

[11] J. Glaunès, M. Vaillant, and M. I. Miller, Landmark matching via large deformation diffeomorphisms on the sphere. Journal of Mathematical Imaging and Vision 20:179-200, 2004, MIA 2002 special issue. MR2049789 (2005b:68275)

[12] A. Guimon, A. Roche, N. Ayache, and J. Meunier. Three-dimensional brain warping using the demons algorithm and adaptive intensity corrections. Technical report, INIRIA Sophia-Antipolis, 1999.

[13] D. D. Holm, Euler-Poincaré dynamics of perfect complex fluids. In P. Newton, P. Holmes, and A. Weinstein, editors, Geometry, Mechanics, and Dynamics: in honor of the 60th birthday of Jerrold E. Marsden, pages 113-167. Springer, 2002. MR1919828 (2003h:37140)

[14] D. D. Holm, J. E. Marsden, and T. S. Ratiu. The Euler-Poincaré equations and semidirect products with applications to continuum theories. Adv. in Math., 137:1-81, 1998. MR.1627802 (99e:58070)

[15] S. Joshi, A. Klassen, E. Srivastava, and I. Jermyn. Removing shape-preserving transformations in square-root elastic (sre) framework for shape analysis of curves. In Springer, editor, Energy Minimization Methods in Computer Vision and Pattern Recognition, EMMCVPR 2007, number 4679 in Lecture Notes in Computer Science, pages 387-398, 2007.

[16] S. Joshi and M. Miller. Landmark matching via large deformation diffeomorphisms. IEEE Transactions in Image Processing, 9(8):1357-1370, 2000. MR.1808275 (2001k:37138)

[17] P. A. Kuz'min, Two-component generalizations of the Camassa-Holm equation. Math. Notes, 81:130-134, 2007. MR2333873 (2008d:37134)

[18] J. E. Marsden and T. S. Ratiu, Introduction to Mechanics and Symmetry. Springer, 1999. MR:1723696 (2000i:70002)

[19] R. McLachlan and S. Marsland. Kelvin-Helmholtz instability of momentum sheets in the Euler equations for planar diffeomorphisms. SIAM J. Appl. Dyn. Sys., 5:726-758, 2006. MR2274496 $(2007 \mathrm{j}: 37132)$

[20] M. I. Miller and L. Younes. Group action, diffeomorphism and matching: A general framework. Int. J. Comp. Vis, 41:61-84, 2001. (Originally published in electronic form in: Proceeding of SCTV 99, http://www.cis.ohio-state.edu/szhu/SCTV99.html). 
[21] W. Mio, A. Srivastava, and S. Joshi. On the shape of plane elastic curves. Technical report, Department of Mathematics, Florida State Univ., 2005.

[22] A. Qiu, L. Younes, and M. I. Miller, Intrinsic and extrinsic analysis in computational anatomy. Neuroimage, 2007. In press.

[23] A. W. Toga, editor. Brain warping. Academic Press, 1999.

[24] A. Trouvé. Diffeomorphism groups and pattern matching in image analysis. Int. J. of Comp. Vis., 28(3):213-221, 1998.

[25] A. Trouvé and L. Younes. Diffeomorphic matching in 1d: Designing and minimizing matching functionals. In D. Vernon, editor, Proceedings of ECCV 2000, 2000.

[26] A. Trouvé and L. Younes. On a class of optimal matching problems in 1 dimension. Siam J. Control Opt., 39(4):1112-1135, 2001. MR1814269 (2002b:49027)

[27] A. Trouvé and L. Younes. Local geometry of deformable templates. SIAM J. Math. Anal., 37(1):1759, 2005. MR2176922 (2006g:58010)

[28] A. Trouvé and L. Younes. Metamorphoses through Lie group action. Found. Comp. Math., pages 173-198, 2005. MR2149415(2006a:68161)

[29] M. Vaillant and J. Glaunès. Surface matching via currents. In Springer, editor, Proceedings of Information Processing in Medical Imaging (IPMI 2005), number 3565 in Lecture Notes in Computer Science, 2005.

[30] M. Vaillant, M. I. Miller, A. Trouvé, and L. Younes. Statistics on diffeomorphisms via tangent space representations. Neuroimage, 23(S1):S161-S169, 2004.

[31] L. Wang, M. F. Beg, J. T. Ratnanather, C. Ceritoglu, L. Younes, J. Morris, J. Csernansky, and M. I. Miller, Large deformation diffeomorphism and momentum based hippocampal shape discrimination in dementia of the alzheimer type. IEEE Transactions on Medical Imaging, 26(462-470), 2006 .

[32] L. Younes. Computable elastic distances between shapes. SIAM J. Appl. Math, 58(2):565-586, 1998. MR.1617630(99c:68221)

[33] L. Younes, P. Michor, J. Shah, and D. Mumford. A metric on shape spaces with explicit geodesics. Rendiconti Lincei - Math. e Appl., 19(1):25-57, 2008. MR.2383560 